\title{
Traffic Analysis of Avatars in Second Life
}

\author{
James Kinicki \\ University of Michigan \\ Ann Arbor, MI 48109, USA \\ kinickij@umich.edu@umich.edu
}

\author{
Mark Claypool \\ Computer Science Department \\ Worcester Polytechnic Institute \\ Worcester, MA 01609, USA \\ claypool@cs.wpi.edu
}

\begin{abstract}
New forms for interaction in virtual worlds, such as Second Life, bring dynamic network traffic because the number of online users can vary greatly from region to region and users can add their own content, such as dynamic objects and custom artwork. While there have been numerous studies on network traffic for multimedia applications and even online games, there has been little effort in understanding the traffic profile for virtual worlds. Earlier work suggests bandwidth used by Second Life varies with the avatar actions and amount of streaming content. Our paper complements this work, first by confirming some earlier results, then by strengthening the network analysis by extending the set of avatar actions studied and by varying the number of objects and avatars interacted with in the virtual world. Our results show that the population and number of objects near an avatar in the virtual world have a dramatic effect on the network characteristics, with dense, crowded areas demanding far more bandwidth than sparse, deserted areas. Similarly, avatar actions that require fast motion, such as walking and flying, use more bandwidth than standing, especially when the former is in a dense area and the latter is in a sparse area. The analysis in this paper can help network planning for access links and core networks as well as provide a base for building synthetic models for simulation.
\end{abstract}

\section{INTRODUCTION}

Virtual worlds are computer-based simulations where users interact with synthetic environments via an avatar. Online, multi-user virtual worlds provide opportunities for people to interact socially from their individual computers located throughout the world. Virtual worlds promise to better support human behavior, provide new forms of human expression, and create opportunities for business and leisure. Companies ranging from Walt Disney to Wells Fargo are developing new virtual worlds that provide interactive experiences suitable for their target demographic audiences [7]. The virtual world Second Life ${ }^{1}$ perhaps the most well-known virtual

$\overline{{ }^{1} \text { http://secondlife.com/ }}$

Permission to make digital or hard copies of all or part of this work for personal or classroom use is granted without fee provided that copies are not made or distributed for profit or commercial advantage and that copies bear this notice and the full citation on the first page. To copy otherwise, to republish, to post on servers or to redistribute to lists, requires prior specific permission and/or a fee.

NOSSDAV'08 Braunschweig, Germany

Copyright 2008 ACM 978-1-60588-157-6/05/2008 ...\$5.00. world today, boasts over 12 million users (called residents), with over 950 thousand having logged in, in from mid-March 2008 to mid-April 2008 month. $^{2}$

While online games have been popular for at least fifteen years, virtual worlds differ from traditional online games in several key ways. In online games, the forms of interaction are limited, typically restricted to gameplay interactions. Virtual worlds, on the other hand, seek to provide flexible forms of interaction, providing for emergent user behavior. Online games have relatively static, preexisting environments that are either fixed by the game developer or customized by the user and loaded once when the game starts. Virtual worlds, conversely, are dynamic, allowing users to add objects, images, video and other features to the world on the fly.

Study of network impact has generally found that online games have low bitrate requirements, sending small but frequent packets in order to synchronize player actions. Large online games can have servers with substantial aggregate traffic, but with game clients that tend to have bandwidth far below the capacities of broadband connections and that are even lower than dialup connections. However, preliminary study of Second Life [6] shows it to use significantly more bandwidth than online games, especially when residents stream music and video in Second Life.

This paper investigates the network characteristics (the size and frequency of data sent and the overall bitrate), which we call turbulence, ${ }^{3}$ for a variety of avatar actions and zones in Second Life.

This paper makes four key scientific contributions in seeking answers to the following questions (with a brief answer, as revealed later in this paper, provided in parentheses):

1. Are the results from previous experiments [6] reproducible? While often overlooked by the computer science community, reproducibility is one of the main principles of the scientific method and refers to the ability of an experiment to be accurately replicated by someone else working independently. It is crucial for experimental results to be reproduced by others within the scientific community in order to generalize the knowledge beyond the experience of the individual scientist. As such, the early results on Second Life must be viewed as tentative until they have been reproduced. (Answer: Yes, in terms of general trends, but specific numbers

\footnotetext{
$\overline{{ }^{2} \text { http://secondlife.com/whatis/economy_stats.php }}$

${ }^{3}$ The term "footprint" is often used in systems work in the context of the basic size a piece of memory of some software. In a network, the size and distribution of packets over time is important, hence our word "turbulence".
} 
are different.)

2. Does the turbulence of Second Life vary with number of objects and/or avatars in a zone? Our experiments expand earlier results by drilling down another step on the effects of zone popularity on network traffic. Results are provided that better isolate the effects of both the density of objects and the population of other avatars on network traffic. (Answer: Yes, both objects and avatars affect turbulence.)

3. What is the turbulence for teleportation, a fundamental avatar action? In addition to standing, walking, and flying, the network impact of an additional resident action namely, teleporting - an action frequently undertaken by residents as they explore the Second Life world is measured. (Answer: Teleportation generally has more turbulence than other actions.)

4. How does the turbulence of Second Life compare with that of online games? The models of interaction in online worlds, where a user manipulates an avatar in order to interact with other avatars and online objects, makes applications such as Second Life most similar to online games. A meta-analysis of previously published results is conducted for online games as a means of better understanding the turbulence of Second Life. (Answer: Second Life has considerably more turbulence than online games.)

The rest of this paper is organized as follows: Section 2 provides some related work on network characterization; Section 3 describes our methodology to characterize the traffic of Second Life; Section 4 analyzes the measured results; and Section 5 summarizes our conclusions and presents possible future work.

\section{RELATED WORK}

Interactions in Second Life are most similar to network games. Overall, online games send small, frequent packets with more traffic sent from the server to the client than from the client to the server. Client to server traffic varies with the frequency of player actions, while the server to client traffic tends to be periodic, but can vary with the action taking place in the world and the number of other players at the server.

First-person shooters, where the player looks through an avatar's eyes and engages in ranged combat with other players, have been heavily studied. Feng et al. analyze traffic from a busy Counter-strike server [5], showing client bandwidth is typically under $56 \mathrm{~kb} / \mathrm{s}$ with periodic small packets. Zander and Armitage analyze and model the traffic characteristics of the Xbox game Halo 2, also showing bandwidth depends upon the number of simultaneous players. Lang et al. show similar analysis for Quake 3 [9].

Massively Multiplayer Online (MMO) games have seen increasing study. Kim et al. present traffic measurements of Lineage II [8], a popular MMO, observing small packets from server to client and even smaller packets from client to server. Bandwidth use from the server is about ten times that from the client and scales linearly with the number of other players. Chen et al. analyze a network trace of ShenZhou Online [2], a prominent game in Asia, providing similar results.

Real-time Strategy (RTS) games have a more indirect form of interaction as the player manipulates the world from a top-down perspective. Claypool characterizes traffic from several popular RTS games [3], showing RTS games basically produce very small, regularly-spaced packets and very modest aggregate bitrates.

Our work differs in that the virtual world analyzed, Second Life, can have more users (about 5-10 times more in one place) than typical FPS or RTS games and, more importantly and unlike even MMO games, allows users to add their own dynamic content in real-time as other users interact with the world.

The only previous known analysis of Second Life (Fernandes et al. [6]) took a first step in profiling the traffic sent to Second Life clients. Their results show that Second Life makes more intensive use of network resources than typical online games, mostly due to external streaming audio. They analyze three avatar actions and two Second Life zones, showing action and popularity both affect network turbulence.

Our work complements theirs by reproducing their results, while extending their work to an additional avatar action and, more significantly, differentiating Second Life zones by the density of the objects and number of other avatars.

\section{METHODOLOGY}

The following methodology was employed to characterize network traffic in Second Life: 1. Determine avatar actions to study (Section 3.1); 2. Select Second Life zones to visit (Section 3.2); 3. Setup measurement environment (Section 3.3); 4. Gather data (Section 3.4); and 5. Analyze results (Section 4). Each step is explained in more depth in the following subsections.

\subsection{Avatar Actions}

Each experiment consisted of a Second Life user performing four different actions with an avatar:

Teleporting - user selects new zone on map and teleports avatar, standing after arrival

Standing - avatar remains still (no movement of mouse or keyboard)

Walking - avatar moves in straight line across single zone at constant speed

Flying - avatar flies in circle around the edge of single zone at constant speed

\subsection{Second Life Zones}

Fernandes et al. [6] chose two different zones to study: a popular zone (Goddess of Love) and an unpopular zone (Menglin II). While it was our intent to reproduce their experiments, unfortunately, neither zone existed any longer at the time of our data collection (about 6 months after their data collection). In addition, Fernandes et al. had not provided a characterization of the number of objects nor the number of other avatars present during their experiments.

Since our hypothesis is that both objects and avatars affect the Second Life network traffic, our aim was to find four zones for our experiments: sparse and deserted, dense and deserted, sparse and crowded, and dense and crowded. This would allow us to evaluate the effects of both objects and avatars on Second Life traffic. However, it turns out that it is unusual for a zone to be sparse and crowded, likely because a zone without objects is typically not interesting enough to draw a crowd. Thus, three zones were settled upon (summarized in Table 1): Isis with a large number of 
objects (dense) and a large population (crowded), Solaris with a large number of objects (dense) with a low population (deserted), and Cyclops with a small number of objects (sparse) with a low population (deserted).

\begin{tabular}{|l|r|r|}
\hline Zone & Objects & Avatars \\
\hline \hline Isis & dense & crowded \\
\hline Solaris & dense & deserted \\
\hline Cyclops & sparse & deserted \\
\hline
\end{tabular}

Table 1: Second Life Zones Selected for this Study

\subsection{Measurement Environment}

For data collection, the client was a PC running Windows XP pro on a $2.8 \mathrm{GHz}$ P 4 with $1 \mathrm{~GB}$ of RAM, with Second Life (v 1.18.2). Based on Fernandes et al. [6], a residential broadband connection is not the bottleneck for a Second Life client. Thus, for convenience, all experiments were collected over a cable modem with a downlink capacity of $4.5 \mathrm{Mb} / \mathrm{s}$ and an uplink capacity of $1 \mathrm{Mb} / \mathrm{s}$. Wireshark ${ }^{4}$ running on the same PC was used to capture all network traffic, whereupon only data to/from the client and Second Life servers was selected for analysis.

\subsection{Data Gathering}

The Second Life architecture has dedicated servers for login, instant messaging, and database functionality. ${ }^{5}$ However, during an extended resident session, the bulk of the network traffic for a client is to and from the simulator processes which run on the primary Second Life servers. Each simulator process manages one $256 x 256$ meter zone, handing off the resident to adjacent simulators as the avatar moves from zone to zone. The simulator stores the state of objects, land parcels, and terrain height-maps for its zone. The simulator makes visibility calculations for each resident, determining what objects and land formations to transmit to the client. Chat and instant messages are also processed by the simulator process. Only data to the simulator processes is considered in subsequent analysis. While Second Life uses TCP for communication with the login server and other utilities, communication between Second Life clients and the server processes use UDP.

Care was taken to not actually leave the zone of study as the avatar moved. With the standing and flying actions, the avatar could circle indefinitely, but with walking, the avatar could proceed from one end of the zone until the far edge was reached. This limited our capture length to about 30 seconds. Teleporting is an instantaneous resident action, but data was selected until the time all new objects on the screen had been rendered.

When data was gathered, Cyclops and Solaris had no other avatars, and zero to two people in adjacent zones, while Isis had between 90 and 100 people during all the captures. While Second Life does support live audio and video streaming, such media is typically served by non-Second Life servers (such as an Internet radio station). Since the focus of our characterization is on the effects of avatars and objects in a virtual world, streaming media was turned off in all experiments.

\footnotetext{
$\overline{{ }^{4} \text { http://www.wireshark.org/ }}$

${ }^{5}$ http://wiki.secondlife.com/wiki/Server_architecture
}

\section{ANALYSIS}

This section begins by analysis of the bandwidth characteristics for each action and each zone (Section 4.1, then drilling down to study packet sizes (Section 4.2) and interpacket times (Section 4.3), both upstream (from client to server) and downstream (from server to client). The section concludes with a brief comparison of the traffic characteristics of Second Life clients with that of online game clients (Section 4.4).

\subsection{Bandwidth}

\begin{tabular}{|c|c|c|c|c|}
\hline \multirow{2}{*}{ Zone } & \multicolumn{4}{|c|}{ Downstream (kbits/s) } \\
\cline { 2 - 5 } & Standing & Walking & Teleport & Flying \\
\hline \hline Dns, Crwd & $192(178)$ & $703(66)$ & $1164(109)$ & $877(169)$ \\
\hline Dns, Dsrt & $141(5)$ & $278(159)$ & $445(111)$ & $821(162)$ \\
\hline Sprs, Dsrt & $10(4)$ & $31(7)$ & $448(103)$ & $27(3)$ \\
\hline
\end{tabular}

Table 2: Downstream Bandwidth - mean (stddev)

\begin{tabular}{|c|c|c|c|c|}
\hline \multirow{2}{*}{ Zone } & \multicolumn{4}{|c|}{ Upstream (kbits/s) } \\
\cline { 2 - 5 } & Standing & Walking & Teleport & Flying \\
\hline \hline Dns, Crwd & $15(2)$ & $31(4)$ & $33(6)$ & $31(7)$ \\
\hline Dns, Dsrt & $30(2)$ & $46(5)$ & $36(7)$ & $52(13)$ \\
\hline Sprs, Dsrt & $13(2)$ & $74(3)$ & $36(25)$ & $73(5)$ \\
\hline
\end{tabular}

Table 3: Upstream Bandwidth - mean (stddev)

Table 2 shows the downstream bandwidth for Second Life for zones that are dense and crowded, dense and deserted and sparse and deserted. Bandwidth is calculated over 500 millisecond intervals. The bandwidth averages and standard deviations are shown for the four actions described in Section 3.1.

Looking first at the actions, mobility (walking and flying) require substantially more bandwidth (about 7-9 times more) than no mobility (standing). However, standing in a dense, crowded area can still have moderate spikes in bandwidth (as high as walking and flying). Flying requires only slightly more bandwidth than walking, even though the apparent speed (to the user) for flying is much greater than for walking. Teleportation also requires considerable bandwidth, about as much as flying.

Looking next at the different zones, dense and crowded zones require more bandwidth than do sparse and deserted zones. However, the latter still require substantial bandwidth when teleporting. In particular, flying in a dense area, even when deserted, requires considerable bandwidth as frequent object data needs to be sent to the client, while bandwidth for walking is considerably more modest when there are fewer avatars, most likely because the slower moving avatar does not receive object data as often.

Table 3 also shows the upstream bandwidth. In general, the upstream bandwidth is about a magnitude less than the downstream bandwidth. Standing still requires less bandwidth than walking and flying, and walking and flying require about the same bandwidth. In addition, there is not as marked a difference in the bandwidth use for the different zones, with sparse/dense and deserted/crowded having little correlation on bandwidth.

Overall, the trends of our results are similar to that in Fernandes et al. [6] in that the downstream bandwidth use 


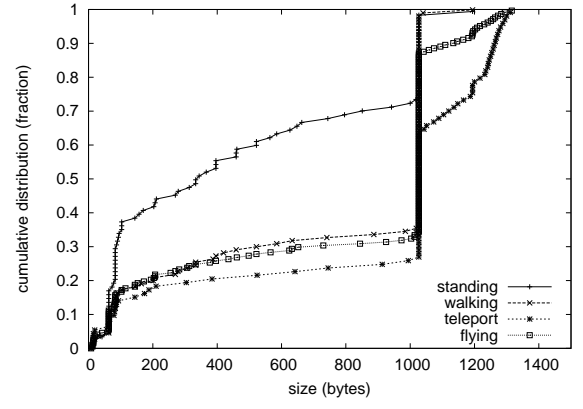

Figure 1: Dense, Crowded

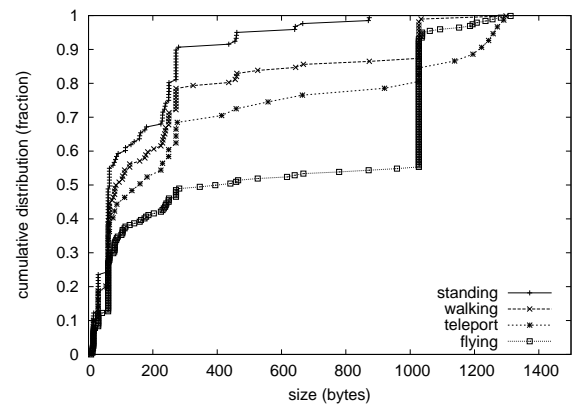

Figure 2: Dense, Deserted

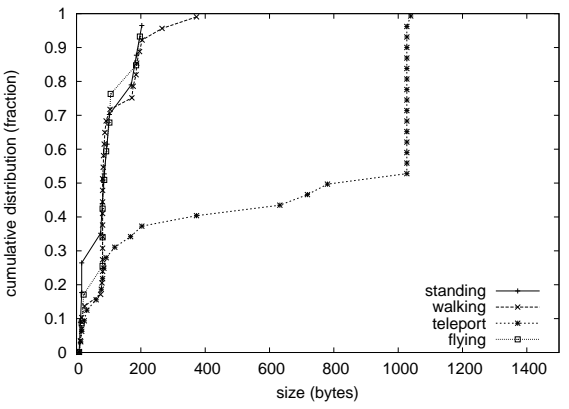

Figure 3: Sparse, Deserted

Packet Size Downstream (bytes)

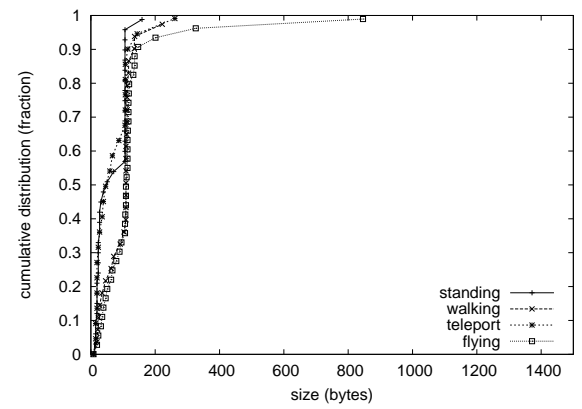

Figure 4: Dense, Crowded

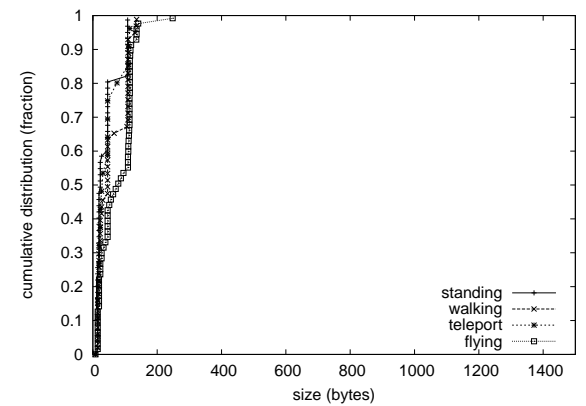

Figure 5: Dense, Deserted

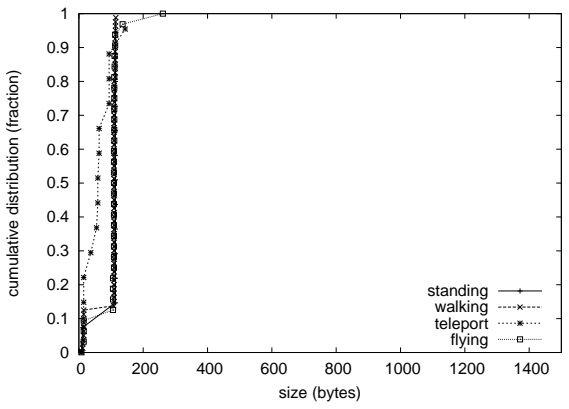

Figure 6: Sparse, Deserted

Packet Size Upstream (bytes) in a dense, crowded zone is considerably higher than in a sparse, deserted zone. However, the magnitude of the difference is greater in our results (7-9 times) than in [6] (2.5 times). This could be because the unpopular area studied by Fernandes et al. had few avatars but some objects, falling in between the sparse-dense range studied here. The bandwidth use for standing, walking and flying are comparable, while our results on teleporting are new.

\subsection{Packet Size}

Figures 1, 2, and 3 depict cumulative distribution functions of the downstream packet sizes for Second Life dense and crowded, dense and deserted and sparse and deserted zones, respectively. The four trendlines are associated with standing, walking, flying and teleporting.

Looking first at the different actions, standing, in general, has the smallest packet sizes, with walking and flying having larger packet sizes. Teleporting has moderately larger packet sizes, even for a spare, deserted zone. There is a noticeable vertical jump in all the graphs at about 1100 bytes, a value considerably smaller than the typical Internet Maximum Transmission Unit (MTU). Next comparing across zones, density and crowded both result in larger packet sizes, while sparse and deserted has small packet sizes, under 300 bytes, for all actions.

Figures 4, 5, and 6 show the corresponding upstream packet size distributions. In general, the upstream packet sizes are uniformly small and mostly independent of avatar action and density/population. There is a noticeable step at around 150 bytes in each distribution.

Overall, the trends of our results are quite similar to those of Fernandes et al. [6] for both upstream and downstream packet size. Our dense and deserted analysis shows downstream packet size distributions in-between the other two areas.

\subsection{Inter-packet Time}




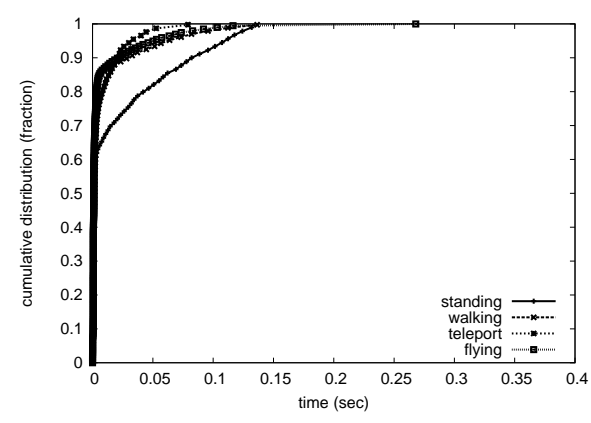

Figure 7: Dense, Crowded

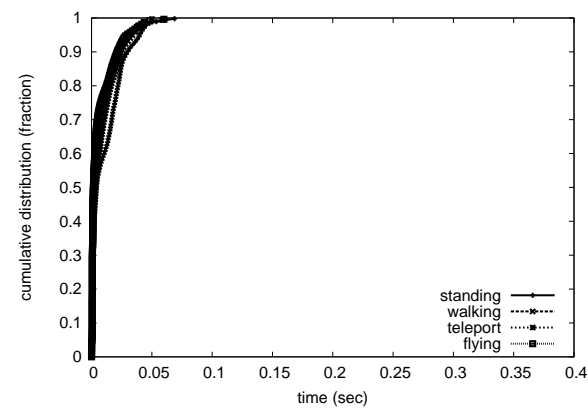

Figure 8: Dense, Deserted

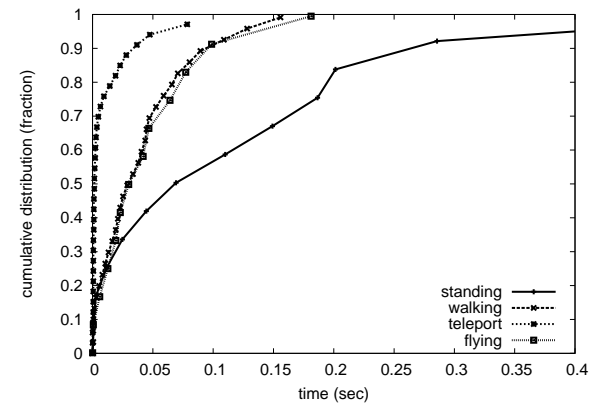

Figure 9: Sparse, Deserted

\section{Inter-packet Time Downstream (milliseconds)}

Figures 7, 8, and 9 depict cumulative distribution functions of the downstream inter-packet times for the three zones and the four actions studied. In general, Second Life servers sends frequent packets, regardless of zone or action, with dense or crowded zones sending at least 20-30 packets per second. Moreover, over half the packets arrive backto-back. For the sparse, deserted zone, standing has a noticeably flatter distribution with many packets arriving at a rate of 3-10 packets per second and flying and walking having mostly 6-12 packets per second. In all cases, packet rates are among the highest for teleporting, with most packets arriving at rate of 30-40 packets per second.

Figures 10, 11, and 12 show the corresponding upstream inter-packet distributions. In general, upstream packet rates are about 20 packets per second, with standing having noticeably lower packet rates for the dense and crowded area and the sparse and deserted area. In the sparse and de- serted area, walking and flying still maintain a high upstream packet rate.

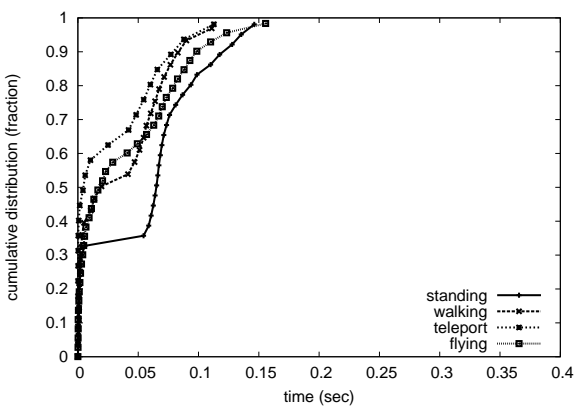

Figure 10: Dense, Crowded

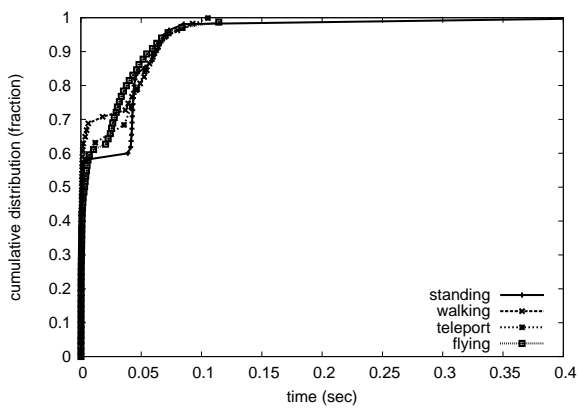

Figure 11: Dense, Deserted

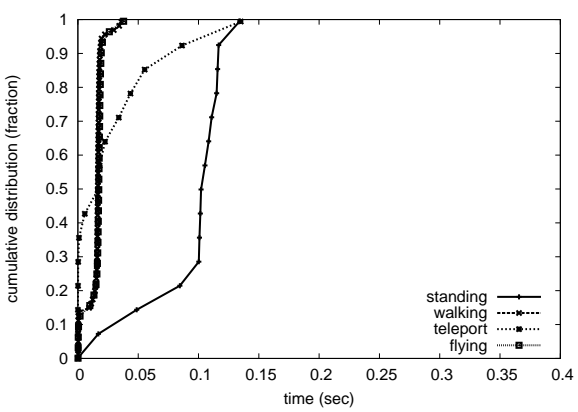

Figure 12: Sparse, Deserted

\section{Inter-packet Time Upstream (milliseconds)}

\subsection{Comparison to Online Games}

In order to better put the network characteristics of Second Life in context, this Section briefly compares the results above with previously published results on turbulence in online games. Based on Claypool and Claypool [4], a broad classification of games can be considered along the lines of perspective and model, resulting in first-person avatar, third-person avatar and third-person omnipresent. Warcraft is chosen as a third-person, omnipresent game, Madden NFL as a third-person, avatar game and Unreal Tournament as a first-person, avatar game. Network traces for each game 
are available online ${ }^{6}$ with published analysis in Sheldon et al. [11], Nichols and Claypool [10] and Beigbeder et al. [1].

A complete comparison for all zones, upstream and downstream, using bandwidth, packet size and inter-packet times is difficult given space constraints and, moreover, such analysis would likely not be informative because of the sheer number of details. Instead, our focus is on downstream traffic, typically the bottleneck, with median behavior for bandwidth use, packet size and inter-packet times. For the online games, general gameplay is used and not specific, isolated player actions. For Second Life, walking is used as the action in a dense, crowded zone, as this is more likely representative of a typical client's network traffic than is traffic in an deserted (hence unvisited) zone.

\begin{tabular}{|l|c|c|c|}
\hline Game & $\begin{array}{c}\text { Bandwidth } \\
(\text { kbits/s) }\end{array}$ & $\begin{array}{c}\text { Pkt Size } \\
\text { (bytes) }\end{array}$ & $\begin{array}{c}\text { Inter-Pkt } \\
(\mathrm{msec})\end{array}$ \\
\hline \hline Warcraft & 5 & 49 & 200 \\
\hline Madden NFL & 14 & 77 & 75 \\
\hline Unreal Tournament & 67 & 75 & 45 \\
\hline Second Life & 775 & 1027 & 9 \\
\hline
\end{tabular}

Table 4: Network Characteristics (median)

Table 4 presents a comparison of the relevant data. Overall, the network turbulence of Second Life is far greater than for other online games. Bandwidth use is 10-100 times greater, packet sizes 15-20 times larger, and packets sent 320 times more often. This reflects the dynamic nature of the environment in Second Life where flexible, custom content created and populated by users must be frequently propagated to the end-host clients. This large turbulence suggests meeting the quality of service requirements of Second Life, which are likely similar to that of third-person avatar games, is a challenge.

\section{CONCLUSIONS}

The growth in popularity of interactive, online environments brings importance to the characterization of their network traffic. The focus of the analysis of this paper is on the bandwidth, packet size and inter-packet times (the turbulence) for Second Life, a popular, online virtual world. The analysis presented can be used to answer the questions posed in the Introduction (Section 1):

1. Previous experimental results published by Fernandes et al. [6] are somewhat reproducible, showing zone and avatar action both have a marked affect on Second Life turbulence. However, the magnitude of the effect on bandwidth observed are different. This suggests further work may be necessary before formal models of bandwidth use in Second Life can be constructed.

2. Bandwidth, packet size and inter-packet times are all impacted by both the number of objects and the number of other avatars in the Second Life zone. Dense, crowded zones have about 10 times the bandwidth and packet size of sparse, deserted zones, while dense, deserted zones have about twice the bandwidth and packet size of sparse, deserted zones. This suggests the number of other avatars in a zone plays a larger role in Second Life turbulence than does the number of other objects in a zone.

\footnotetext{
${ }^{6} \mathrm{http}: / /$ perform.wpi.edu/downloads/
}

3. Teleportation, when an avatar first jumps to a new zone, has considerable turbulence, with bandwidth, packet size and inter-packet times comparable to those of the most demanding actions. This holds for all zones, regardless of object density or population of other avatars.

4. Second Life has much more turbulence than online games, with about 10 times the bandwidth and packet size of games of all genres.

The reported data rates for a Second Life client can help users in broadband selection choices, and when aggregated over a projected number of users can help ISPs with capacity planning. Packet sizes and inter-packet times can be used in classification of Second Life traffic as well as provide a beginning for building synthetic models for simulation.

Second Life allows users to buy their own private island, giving full control over access to the land and having the land zone hosted on a dedicated server process. Such a setup would provide an environment in which the exact number of objects and guests could be controlled. Future work could proceed with an experimental methodology similar to that in this paper, but using this controlled environment.

Second Life clients cache textures, which may constitute a major percentage of data transmitted. Future work could explore how caching affects turbulence. Similarly, analysis could help understand the impact of an avatars motion path on turbulence, further helping explain communication between client and server.

Second Life uses a third-person avatar model for player interaction and is thus likely to have similar latency requirements as third-person online games. Future work could do controlled studies with induced latency, examining the effects of latency on users in Second Life.

\section{REFERENCES}

[1] T. Beigbeder, R. Coughlan, C. Lusher, J. Plunkett, E. Agu, and M. Claypool. The Effects of Loss and Latency on User Performance in Unreal Tournament 2003. In NetGames, Portand, OG, USA, Sept. 2004.

[2] K.-T. Chen, P. Huang, C.-Y. Huang, and C.-L. Lei. Game Traffic Analysis: an MMORPG Perspective. In NOSSDAV, Stevenson, WA, USA, June 2005.

[3] M. Claypool. The Effect of Latency on User Performance in Real-Time Strategy Games. Comp. Networks, 49(1), 2005.

[4] M. Claypool and K. Claypool. Latency and Player Actions in Online Games. Comm. of the ACM, 49(11), 2006.

[5] W. Feng, F. Chang, W. Feng, and J. Walpole. Provisioning On-line Games: A Traffic Analysis of a Busy Counter-Strike Server. In IMW, France, Nov. 2002.

[6] S. Fernandes, C. Kamienski, D. Sadok, J. Moreira, and R. Antonello. Traffic Analysis Beyond This World: the Case of Second Life. In NOSSDAV, Illinois, USA, June 2007.

[7] International Digital Software Association. Beyond Second Life, June 112001.

[8] J. Kim, J. Choi, D. Chang, T. Kwon, Y. Choi, and E. Yuk. Traffic Characteristics of a Massively Multiplayer Online Role Playing Game and Its Implications. In NetGames, Hawthorne, NY, USA, Oct. 2005.

[9] T. Lang, P. Branch, and G. Armitage. A Synthetic Traffic Model for Quake 3. In ACE, Singapore, June 2004.

[10] J. Nichols and M. Claypool. The Effects of Latency on Online Madden NFL Football. In NOSSDAV, Kinsale, County Cork, Ireland, June 2004.

[11] N. Sheldon, E. Girard, S. Borg, M. Claypool, and E. Agu. The Effect of Latency on User Performance in Warcraft III. In NetGames, Redwood City, CA, USA, May 2003. 